\title{
Microstrip Diplexers with Dual-mode Patch Resonant Junctions
}

\author{
Eugene A. Ogbodo, Yun Wu, Yi Wang \\ Engineering Science \\ University of Greenwich (Medway campus), \\ Chatham Maritime, Kent, U.K. \\ e.a.ogbodo@gre.ac.uk; y.wu@gre.ac.uk; yi.wang@gre.ac.uk
}

\begin{abstract}
A conventional diplexer is made up of two channel filters which are connected to a transmission-line based signal distribution network. In this work, a novel diplexer has been proposed and designed using hairpin resonator filters that are joined together using a patch resonator. The patch being a dualmode is made to resonate at the centre frequencies of the channel filters in corresponding modes. The patch functions as a signal splitter and at the same time as a resonant pole for each of the channel filters. A diplexer with the channel centre frequencies of 3.99 $\mathrm{GHz}$ and $4.39 \mathrm{GHz}$ and $4 \%$ fractional bandwidth has been designed and tested. The simulated and measured results show very good agreements.
\end{abstract}

Keywords-Diplexer, Patch resonator; Dual-mode resonator

\section{INTRODUCTION}

Dual mode microstrip resonators are playing an important role in the miniaturisation of microwave planar filters. Its popularity comes from its ability to take the place of two single mode resonators, thereby resulting in halving the number of required resonators for a given purpose. In the design of planar filters, microstrip resonators such as patch resonators are used extensively. In [1] a patch resonator was used to generate a transmission zero in a bandpass filter (BPF) response without resorting to any cross couplings, thereby resulting in improved frequency selectivity. Square patch resonators were applied in the design of BPFs in [2] and [3], whereas they were used to design dual-band BPFs in [4] and [5]. A particular area where the use of dual-mode or multi-mode resonators can be beneficial is in diplexer designs with resonant junctions [6]. Conventionally, channel filters in the diplexers are combined via transmission-line based junctions, as illustrated in Fig. 1(a), such as T-junctions, hybrid couplers, manifolds or circulators [7], [12] - [15]. However, the new diplexer configuration based on resonant junctions has attracted a lot of attention in the past few years. Different circuit techniques have been used. For instance, in [8] two novel multiplexer topologies based on allresonator structures were reported at X-band using waveguide technology. In [9], a frequency division duplex (FDD) diplexer that integrates a T-shaped resonator and two sets of open-loop resonators was developed. The technique used in [9] was then extended into a multiplexer and a switchable diplexer in [10] using open-loop resonators. In [11], a compact diplexer was proposed and designed using a stepped-impedance resonator (SIR) which acted as a common resonator.

In this paper, a novel diplexer structure has been proposed and designed using hairpin (HP) resonator filters with a $4 \%$ fractional bandwidth each at $3.99 \mathrm{GHz}$ and $4.39 \mathrm{GHz}$ respectively. These channel filters are linked together using a dual-mode patch resonator. Each of the channels was individually designed using a three-pole structure with proximity couplings. Using dual-mode patch resonator led to the reduction in the number of required resonators from a supposedly six resonators to five resonators. Fig. 1(b) illustrates the resonator topology and the coupling path of the proposed design as compared with the conventional topology shown in Fig. 1(a). The proposed diplexer layout is displayed in Fig. 2.

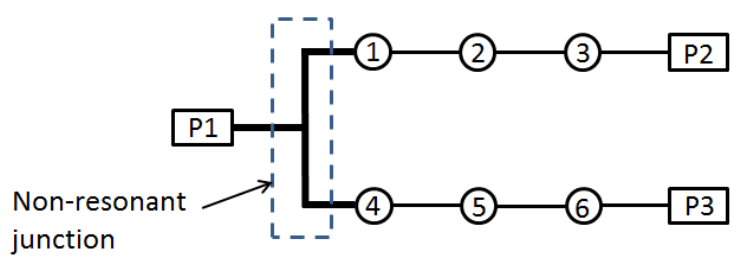

(a)

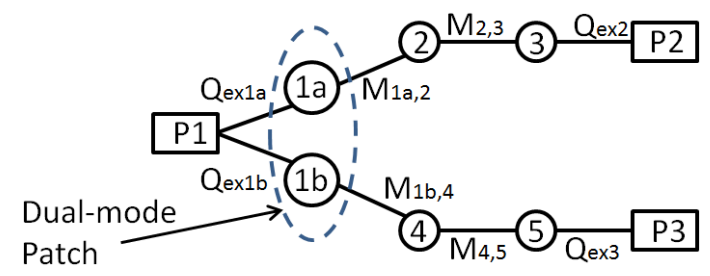

(b)

Fig. 1. (a) A conventional diplexer topology with a non-resonant junction; (b) The proposed diplexer topology wth a resonant dual-mode junction.

\section{DESIGN}

The proposed microstrip diplexer has been designed to meet the following specifications: 
- Center frequency of the lower and upper passbands, $f_{0}, \mathrm{BPL}$ and $f_{0}, \mathrm{BPU}: 3.99 \mathrm{GHz}$ and $4.39 \mathrm{GHz}$

- Passband return loss: $20 \mathrm{~dB}$

- $\quad$ Fractional bandwidth (FBW) of the lower and upper passband, BWL and BWU: $4 \%$

All the simulations in this work were performed using EM simulator Sonnet Suites.

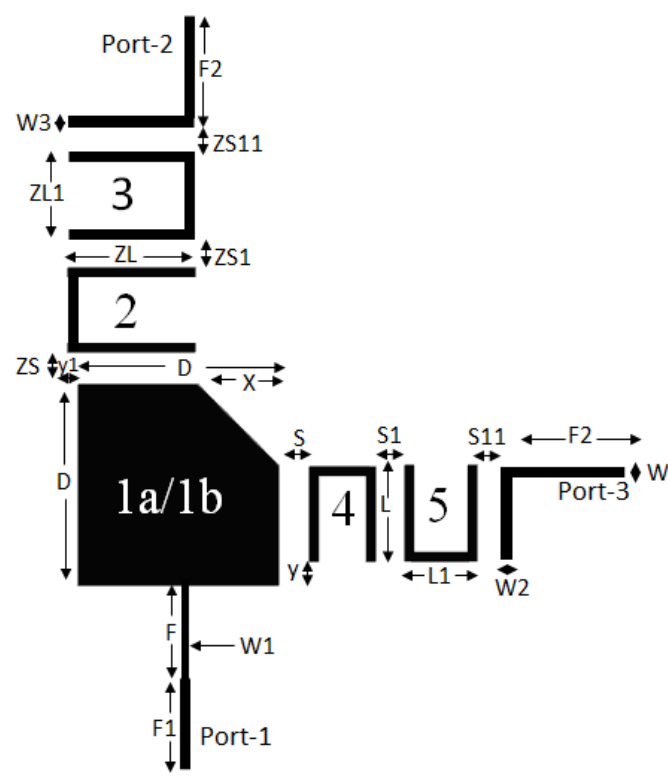

Fig. 2. Layout of the proposed diplexer (unit: $\mathrm{mm}$ ); $\mathrm{ZL}=5.4, \mathrm{ZL} 1=3.9, \mathrm{ZS}=$ $1, \mathrm{ZS} 1=1.4, \mathrm{ZS} 11=0.2, \mathrm{~L}=5, \mathrm{~L} 1=3.4, \mathrm{~S}=0.84, \mathrm{~S} 1=1.26, \mathrm{~S} 11=0.2, \mathrm{D}=$ $10.9, \mathrm{X}=4.6, \mathrm{~F}=6, \mathrm{~F} 1=5, \mathrm{~F} 2=10, \mathrm{~W}=1.1, \mathrm{~W} 1=0.9, \mathrm{~W} 2=0.6, \mathrm{~W} 3=0.9$, $\mathrm{y}=0.5, \mathrm{y} 1=1.6$.

\section{A. Topology and coupling matrix}

As shown in Fig. 1(b), the junction resonator has dual modes, denoted as $1 \mathrm{a}$ and $1 \mathrm{~b}$. The mode-1a is coupled to the resonator-2 and 3 to generate the lower channel, whereas the mode- $1 \mathrm{~b}$ is coupled to the resonator- 4 and 5 to generate the upper channel. Each channel filter was designed separately to have a passband ripple factor of $0.043 \mathrm{~dB}$ using a three-pole Chebychev lowpass prototype derived from [16] with $g$ values of $g_{0}=g_{4}=1.0, g_{1}=g_{3}=0.8516$ and $g_{2}=1.1032$. (1) and (2) are the required external quality factor $Q_{\mathrm{ex}}$ and coupling coefficients used for the physical dimensioning of the microstrip resonators and filters

$$
\begin{gathered}
M_{1 a, 2}=M_{2,3}=M_{1 b, 4}=M_{4,5}=\frac{F B W}{\sqrt{g_{1} g_{2}}}=0.041 \\
Q_{e x 1 a}=Q_{e x 1 b}=Q_{e x 2}=Q_{e x 3}=\frac{g_{1} g_{0}}{F B W}=21.29
\end{gathered}
$$

\section{B. Patch resonator}

The patch resonator is used as the junction resonator in this design. The patch, of dual modes, acts as a resonant pole to each channel filter as well as a signal splitter in the diplexer. A half-wavelength square patch resonator is first made to resonate at the centre frequency of the diplexer. In other to generate the dual modes, a chamfer defined by the dimension
$\mathrm{X}$ is introduced at one corner of the square resonator. By varying $X$, the dual-modes of the patch can be adjusted to the desired frequencies. Fig. 3 illustrates the resonant frequencies of the patch as a function of the chamfer length, $\mathrm{X}$. When $\mathrm{X}$ is $4 \mathrm{~mm}$, the resonance was split into $3.99 \mathrm{GHz}$ and $4.39 \mathrm{GHz}$.

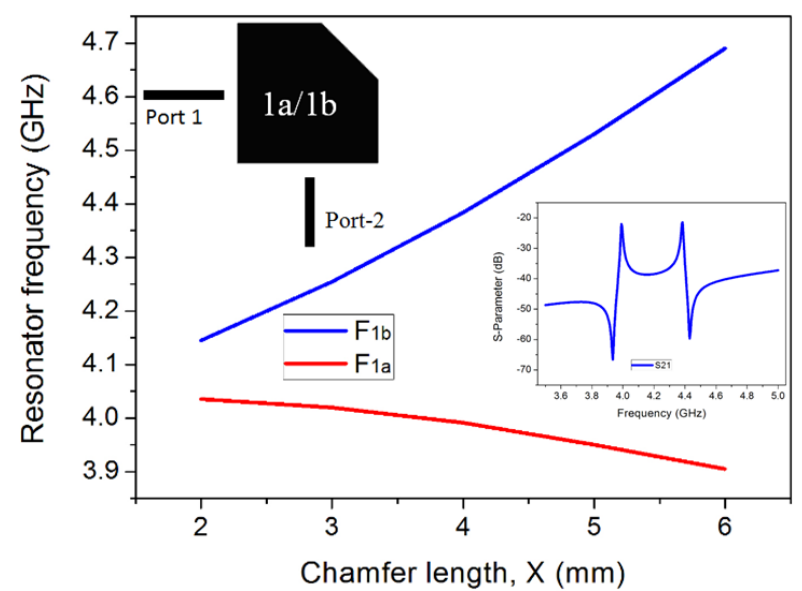

Fig. 3. Two resonance frequencies of the patch as a function of the chamfer length. The insets shows the layout used in th simultion and a typical simultaed $\mathrm{S}_{21}$ response.

\section{Couplings}

To obtain the external quality factor $\left(Q_{\text {exla }}\right.$ and $\left.Q_{\text {exlb }}\right)$ at the common port, a configuration as shown in Fig. 4 was used. At port-1, a feeder line was tapped to the patch resonator whereas port- 2 and port- 3 were weakly coupled to the patch. The feed line of port-1 was adjusted both in length and width. Using (3), the $Q_{\text {ex }}$ towards both modes of the patch was obtained

$$
Q_{e x}=\frac{f_{0}}{\Delta f}
$$

where $\Delta f$ is the 3 -dB bandwidth measured from the peak of the resonance curve. Fig. 4 shows the typical resonance curves between port- 1 and 2 and between port- 1 and 3 .

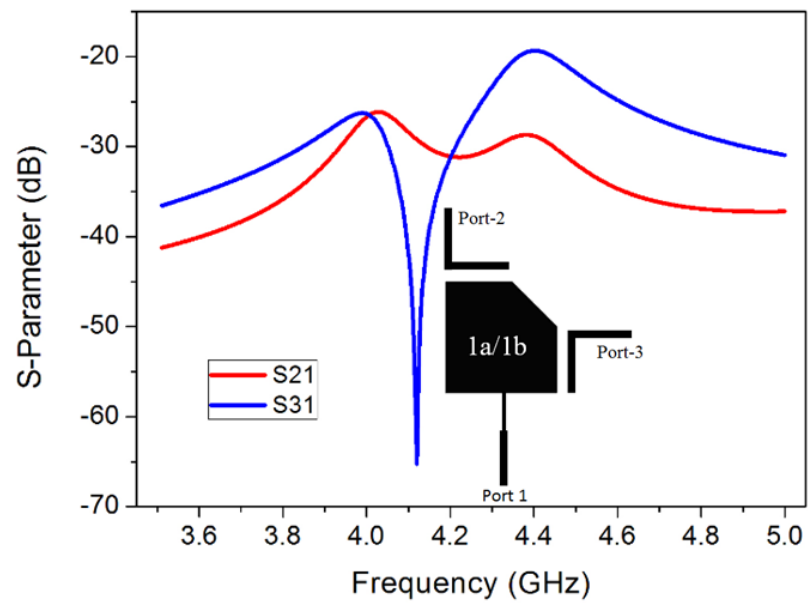

Fig. 4. Configuration used to extract $Q_{\mathrm{ex} 1 \mathrm{a}}$ and $Q_{\mathrm{ex} 1 \mathrm{~b}}$ and the typical resonance curves. 
The coupling coefficients, $M_{1 \mathrm{a}, 2}$ and $M_{1 \mathrm{~b}, 4}$, were extracted by varying the spacing, $\mathrm{ZS}$, between the patch and resonator 2 and $\mathrm{S}$ between the patch and resonator 4 respectively. The layout configurations and the graphs of the coupling coefficient against ZS and S were plotted in Fig. 5(a) and (b). (4) was used to calculate the coupling coefficients. After obtaining the coupling coefficients around the patch separately, resonator 1,2 and 4 were assembled together to evaluate the loading effect of the third port on the coupling. The two pairs of resonance peaks are shown in Fig. 6. It was observed that the interactive effect is not significant. These peaks can also be used in obtaining the coupling coefficients.

$$
M_{1 a, 2}=M_{2,3}=M_{1 b, 4}=M_{4,5}=\frac{f_{2}^{2}-f_{1}^{2}}{f_{2}^{2}+f_{1}^{2}}
$$

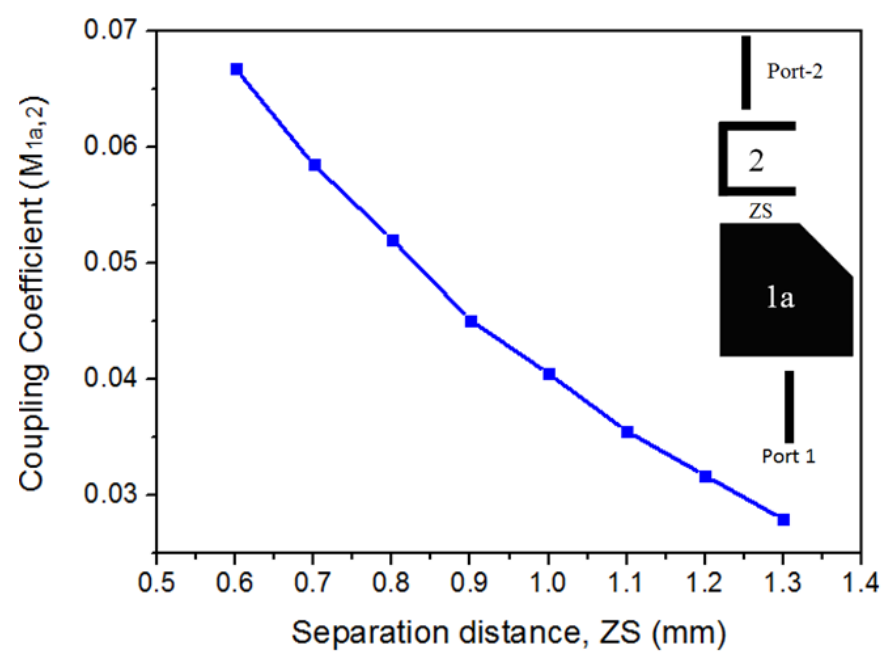

(a)

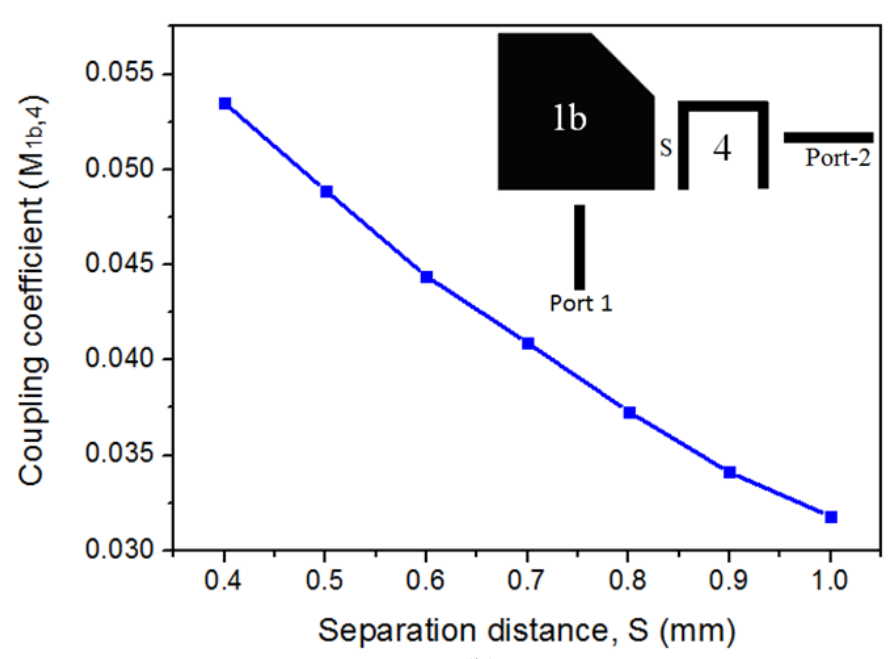

(b)

Fig. 5. (a) Coupling coefficient $M_{1 \mathrm{a}, 2}$ against $\mathrm{ZS}$ and (b) coupling coefficient $M_{1 \mathrm{~b}, 4}$ against $\mathrm{S}$.

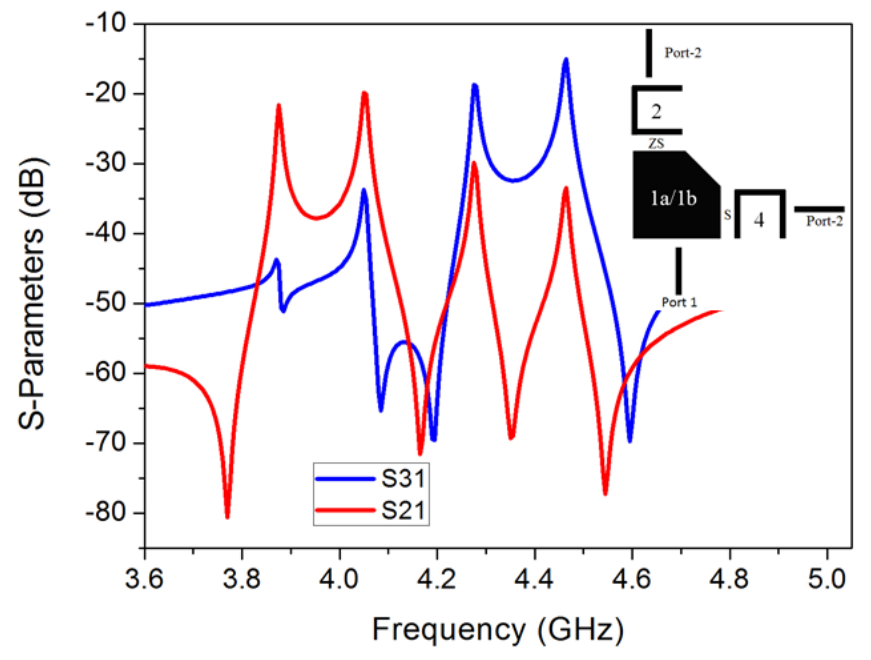

Fig. 6. Simulated resonance curves from the assembled resonator 1, 2 and 4. The inset shows the layout used in the simulation.

\section{Diplexers}

After extracting the coupling coefficients and the $Q_{\text {ex }}$, the diplexer was arranged as shown in Fig. 2 and optimized to specification. Fig. 7 shows the simulated response using dashed lines. Fig. 8(a) and (b) shows the current distributions when the diplexer operates at $3.99 \mathrm{GHz}$ and $4.39 \mathrm{GHz}$. It can be seen that the currents are excited in orthogonal directions on the patch at different frequencies, which means the patch resonator splits the signal into two channels as proposed.

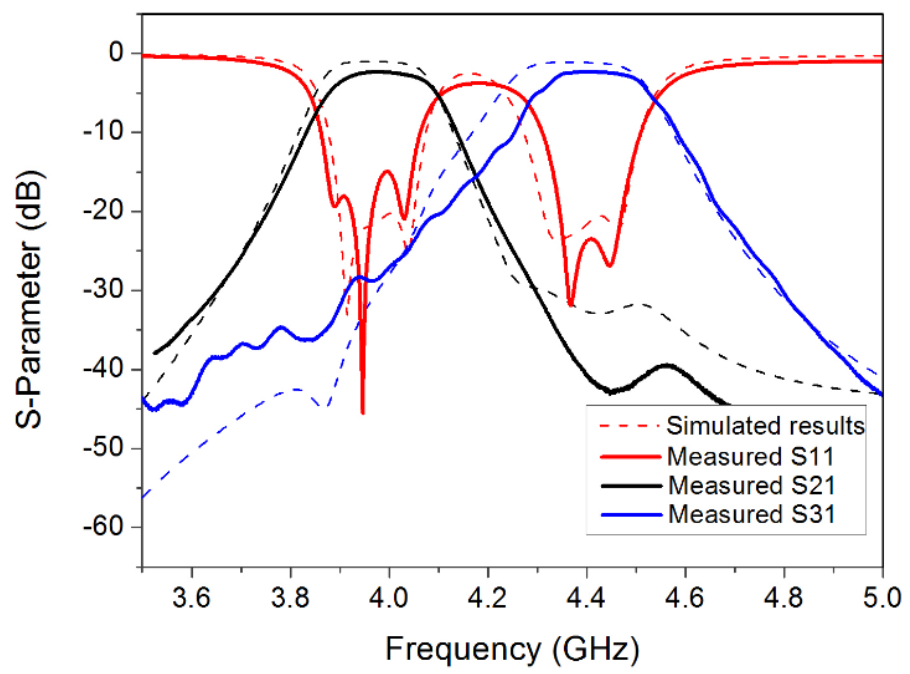

Fig. 7. Measured responses in comparison with full-wave simulations.

\section{FABRICATION AND MEASUREMENTS}

The diplexer was made on a Rogers 3010 substrate with a thickness of $1.27 \mathrm{~mm}$, a relative permittivity of 10.8 and a loss tangent of 0.0022. It was fabricated using LPKF ProtoMat S63 
micro milling process and displayed in Fig. 9. Agilent Network Analyser N5230A was used for measurement. A comparison between the simulated (dash lines) and measured (thick lines) results are shown in Fig. 7. It can be seen that a reasonably good agreement is achieved at both passbands with the return loss measured at about $15 \mathrm{~dB}$. Due to the machining tolerance, the measured results can be seen shifted to the higher frequency by approximately $5 \mathrm{MHz}$. The minimum measured insertion loss in the passband is less than $2 \mathrm{~dB}$. The high passband has a reduced bandwidth.

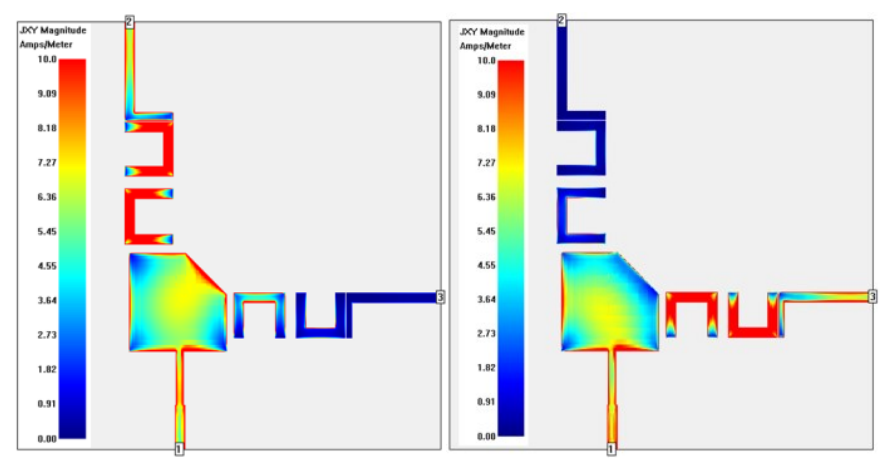

Fig. 8. (a) Current distribution at $3.99 \mathrm{GHz}$ (b) current distribution at $4.39 \mathrm{GHz}$.

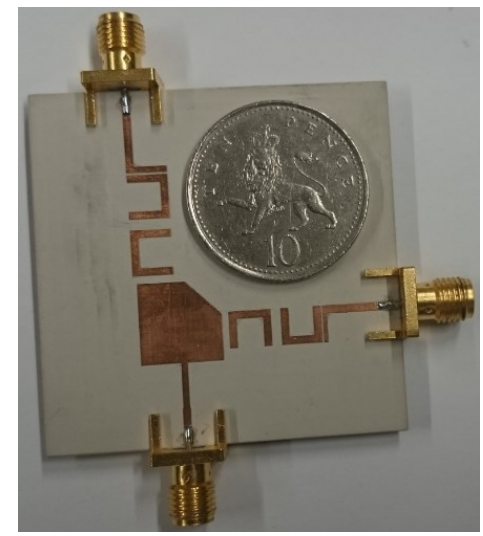

Fig. 9. Photograph of the fabricated diplexer with a dual-mode resonant junction.

\section{CONCLUSION}

In this diplexer design, a patch resonator was used as a signal splitter as well as a resonant pole for each channel filter. The patch was used in joining two three-pole hairpin BPFs operating individually at the required passbands. The patch being a dual-mode replaced two resonators, one from each channel filter. This led to the circuit miniaturization by reducing the number of required resonators and removing the transmission line junction. The passband responses from the measurements and simulations have shown a good agreement, verifying the feasibility of using a dual-mode resonant junction in the diplexer design. Another potential advantage of using the patch resonator is the exploration of other design variations with the patch and parasitic structures in improving the isolation and rejection performance of the diplexer.

\section{REFERENCES}

[1] E. A. Ogbodo, Y. Wang, and P. Rapajic, "Bandpass Filters with Mixed Hairpin and Patch Resonators", Progress In Electromagnetics Research C, Vol. 59, 101-106, 2015.

[2] R. Romero and O. Frazao, "Stopband-Improved Dual-Mode Bandpass filter Using Side-Slit Patch Resonator," Microwave and Optical Technology Letters, Vol. 49, No. 3, March 2007.

[3] Y. M. Bo, X. D. Huang, and C. H. Cheng, "A Novel Bandpass Filter Using A resonator with Combined CPW and Microstrip Input/Output," Microwave and Optical Technology Letters / Vol. 48, No. 11, November 2006.

[4] J.-X. Chen, Z. Xiu-Yin and Q. Xue, "Dual-Band Bandpass Filter and Diplexer Based on Double-sided Parallel-Strip Line", IEEE MTT-S International Microwave Object Identifier, 2008, pages 675-678.

[5] S. Fu, B. Wu, J. Chen, S-J, Sun and C-h Liang, "Novel Second-Order Dual-Mode Dual-Band Filters Using Capacitance Loaded Square loop Resonator," IEEE Transactions on Microwave Theory and Techniques, Vol. 60, No. 3, March 2012.

[6] X. Shang, Y. Wang, W. Xia and M. J. Lancaster, "Novel Multiplexer Topologies Based on All-Resonator Structures," IEEE Transactions on Microwave Theory and Techniques, Vol. 61, No. 11, November 2013.

[7] R. Cameron, M. Yu, "Design of manifold-coupled multiplexers," IEEE Microwave Magazine, vol.8, no.5, pp.46-59, 2007

[8] X. Shang, Y. Wang, X. Wenlin, M.J. Lancaster, "Novel Multiplexer Topologies Based on All-Resonator Structures," IEEE Trans. Microwave Theory and Techniques, vol 61, no. 11, 2013, pp. $3838-$ 3845 .

[9] M. L. Chuang and M. T. Wu, "Microstrip Diplexer Design Using Common T-Shaped Resonator," IEEE Microwave and Wireless Components. Letters, Vol. 21, No. 11, Nov. 2011.

[10] M. L. Chuang and M. T. Wu, "Microstrip Multiplexer and Switchable Diplexer with Joint T-Shaped Resonator," IEEE Microwave and Wireless Components. Letters, Vol. 24, No. 5, May 2014.

[11] C. F. Chen, T. Y. Huang, C. P. Chou and R. B. Wu, "Microstrip Diplexers With Common Resonator Sections for Compact Size, But High Isolation," IEEE Transactions on Microwave Theory and Techniques, Vol. 54, No. 5, May 2006.

[12] T. Yang, P-L. Chi and T. Itoh, "High Isolation and Compact Diplexer Using the Hybrid Resonators," IEEE Microwave and Wireless Components. Letters, Vol. 20, No. 10, Oct. 2010.

[13] C. Chen, H. Wu and W. Wu, "High Isolation DBR Diplexer Using InLine SCMRC", Progress In Electromagnetics Research C, Vol. 22, 97 108, 2011.

[14] T.F. Skaik, M.J. Lancaster, F. Huang, "Synthesis of multiple output coupled resonator circuits using coupling matrix optimisation," Microwaves, Antennas \& Propagation, IET , vol.5, no.9, pp.1081-1088, June 272011.

[15] S. Bastioli, L. Marcaccioli and R. Sorrentino, "An Original Resonant Yjunction for compact Waveguide Diplexers," Proceeding of IEEE MTTS International Microwave Symposium Digest, Boston, 7-12 June 2009, pp. 1233-1236.

[16] J.-S. Hong "Microstrip Filters for RF/Microwave Applications," John Wiley \& Sons, Inc. 2011. 